\title{
Optimal Location of Small Generators in Weak Networks with Optimal Operation
}

\author{
J.A. Domínguez, J.M. Yusta, A.A. Bayod, J.L. Bernal, M.J. Velilla, J. Mur, M.A. García, A. Diaz ${ }^{1}$ \\ ge3 - CIRCE - Department of Electrical Engineering \\ University of Zaragoza \\ C / María de Luna 3, 50018 Zaragoza (Spain) \\ phone: +34976 762 401, fax: + 34976761929 \\ e-mail: jadona@unizar.es,jmyusta@unizar.es, aabayod@unizar.es,jlbernal@unizar.es,mvelilla@unizar.es, \\ joako@unizar.es, magargar@unizar.es \\ ${ }^{1}$ Department of Electrical Engineering \\ UNEXPO University \\ Urbanización Villa Asia - Puerto Ordaz (Venezuela) \\ e-mail: amdiaz@cantv.net
}

\begin{abstract}
This paper presents an algorithm to optimal location of small generators that allows to consider the optimal operation of the distribution network.

An optimal operation of the weak netwoks allows to improve the penetration of distributed generators in the above mentioned netwoks and to improve the benefits of its installation. The used algorithm is based on the heuristic technique of Tabu Search.

The obtained results allow to check the efficiency of the utilised search technique and the benefits that supposes for the location of new generators the optimal operation of the network.
\end{abstract}

\section{Key words}

Distribution Power Systems, Tabu Search, Distributed Generation.

\section{Introduction}

The new generation technologies make efficient and profitable the electric power production to smaller scale. Wind generators, heat and electric power generators, or hydroelectric mini-central give power to the distribution nets in points different from the traditional ones. Some authors [1] surveys the operational and economical benefits of implementing DG's in the distribution network.

Others authors have approached the problem of the dispersed generation planning in distributions networks [2] - [7].

The authors [2] and [3] model the mathematical problem of the network expansion with dispersed generation. The paper [3] presents a new approach to dispersed generation planning based on Hereford ranch algorithm. The benefit is the minimisation of losses.

Some [4] uses a methodology based on an economic optimisation tool which evaluates a range of different options for distributed generation. Then, the author applies technical software tools to consider the effects on energy utilisation and power quality.
The authors [5] reviews the position of distributed generation with respect to the installation and interconnection of such units with the classical grid infrastructure. In particular, the status of technical standards both in Europe and USA, and possible ways to improve the interconnection situation.

Paper [6] exploits the benefits of the distributed generation in the expansion investment plan of a distribution utility. He analyses demand-side resource options using feeder-and time-specific marginal costs.

Several authors [7] use a simulation tool to analyse an assessment of business opportunities to invest in distributed generation. DG success depends on the location of adequate sites to strategically establish few DG units being a substitute to network expansion.

In this paper, we present the incorporation the optimal operation in the dispersed generators planning of renewable energies. The location of these small generators can influence in the network operation. The simulation allows to see the effect that produces a dispersed generator in a network under some certain operation conditions. The optimisation allows to rehearse automatically different operation possibilities and to compare them.

The algorithm, that here is presented, has been developed for the distribution systems and their special ones characteristic. It is based on Tabu Search [8]. It allows to consider the non linear problem by means of a model based on injection of currents, it considers PV-nodes to model the dispersed generators.

\section{Mathematical model}

Mathematically, the model consists on minimizing a non linear objective function $f$ subject to certain restrictions.

Minimise: $f(x, w)$

Subject to:

$$
g(x, w)=0
$$


$h(x, w) \leq 0$

where:

$w$ is the vector of independent variables of control and decision that includes the active power and voltage in the generators $\left(P_{g}, V_{g}\right)$, the regulation taps in the transformers (transformation relationship $t$, and relationship of angles $\delta$.), the steps connected in the capacitors bank $\left(Q_{c}\right)$, the connection or disconnect of small generators and/or controlled loads $\left(y_{l}\right)$, connection or disconnect of generators $\left(y_{g}\right)$.

$x$ is the vector of dependent variables that includes the voltage in PQ nodes, and the reactive power given by the generators and PV nodes.

$f(x, w)$ is the objective function to optimize, it represents the generation cost with penalizations due to the overload of branches and overvoltages or subvoltages in the buses.

$g(x, w)$ are the nodal restrictions due to the power flow.

$h(x, w)$ are the inequations in dependent and independent variables due to the operation limits of the generating devices, the thermal limits of the lines and transformers, and the quality demanded to the profile of tensions (acceptable margins).

\section{A. Evaluation of the solutions with the objective function.}

In this case the objective function $f(x, w)$ is the generation cost with penalization by overload in the feeders and penalization by over and sub-voltages in the buses.

$$
\begin{gathered}
f=\sum_{i \in G} f_{\text {gen }, i}+\sum_{j \in F} f_{\text {rama }, j}+\sum_{k \in B} f_{\text {bus }, k} \\
f_{\text {gen }, i}=c_{f i} \cdot y_{g i}+c_{v i} \cdot P_{g i} \\
f_{\text {rama }, j}=\left\{\begin{array}{cc}
a \cdot\left(i_{\text {actual }, j}-i_{\text {max }, j}\right)^{n} & i_{\text {actual }, j}>i_{\text {max }, j} \\
0 & i_{\text {actual }, j} \leq i_{\text {max }, j}
\end{array}\right. \\
f_{\text {bus }, k}=\left\{\begin{array}{cc}
a \cdot\left(u_{\text {actual }, k}-u_{\text {max }}\right)^{n} & u_{\text {actual }, k}>u_{\text {max }} \\
0 & u_{\text {min }} \leq u_{\text {actual }, k} \leq u_{\text {max }} \\
a \cdot\left(u_{\text {min }}-u_{\text {actual }, k}\right)^{n} & u_{\text {actual }, k}<u_{\text {min }}
\end{array}\right.
\end{gathered}
$$

where:

$f_{\text {gen, } i} \quad$ cost associated to the generator $i \in G$ (set of generators in the net),

$f_{\text {rama }, j}$ penalty cost associated to overload of the branch $j \in R$ (set of branches in the net),

$f_{\text {bus }, k} \quad$ penalty cost associated to sub- and over-voltage in the bus $k \in B$ (set of buses in the net),

$c_{f i}$, fixed cost associated to the operation of the generator $i$,

$y_{g i} \quad$ variable binary that represents the switch on/off of the generator $i$,

$c_{v i} \quad$ variable cost associated to the power supplied by generator $i$,

$P_{g i} \quad$ power supplied by the generator $i$, $i_{a c t u a l, j} \quad$ current that circulates for the branch $j$,

$i_{\text {max }, j} \quad$ maximum current allowed in the branch $j$,

$a, n \quad$ constants that permit regulates the grade of importance that you give to the penalizations,

$u_{\text {actual }, k}$ current voltage in the bus $k$,

$u_{\min } \quad$ minimum voltage allowed in the buses,

$u_{\max } \quad$ maximum tension allowed in the buses.

\section{B. Technical constraints.}

Technical constraints are fundamentally of two types: operation constraints and constraints of the control variables.

Inside the operation constraints are: power limits of feeders and transformers, and margins of the voltage profile. These constraints have been considered with the penalty of the function objective (4) and (5).

The constraints of the control variables determine the values that can take the above mentioned variables and delimit the search space. The following restrictions have been considered:

- operation limits of each generator $i$,

$P_{g_{-} \min }^{i} \geq P_{g i} \geq P_{g_{-} \max }^{i}$

$Q_{g_{-} \text {min }}^{i} \geq Q_{g i} \geq Q_{g_{-} \max }^{i}$

- operation limits of each capacitors bank $i$,

$Q_{c_{-} \min }^{i} \geq Q_{c i} \geq Q_{c_{-} \max }^{i}$

- limits of the control parameters of each transformer $i$,

$t_{\min }^{i} \geq t_{i} \geq t_{\max }^{i}$

$\delta_{\min }^{i} \geq \delta_{i} \geq \delta_{\max }^{i}$

\section{Tabu Search algorithm}

The Tabu Search belongs to the search methods for neighborhoods. These methods start from a feasible initial solution and, by means of modifications of this solution, are obtaining other feasible solutions of its environment. While a certain criterion of stop is not fulfilled, Its store like ideal the best of the visited solutions. Inside these methods, we prune to include the genetic algorithms and the tabu search. Along the search, it allows to visit one another worse solutions that the previous ones, in an intent of diversifying the search. In the same line, it forgives to repeat the movements carried out lately by means of the tabu list. This simple strategy avoids to fall in local minima when diversifying the search.

\section{A. Description of Tabu search.}

A solution of our problem comes defined by a vector $s_{i}=$ $\left\{w_{1}, w_{2}, \ldots, w_{n-l}, w_{n}\right\}$ with the values of the variables independent from control and decision, $w_{k}$. Every modification of the value of a variable $w_{k}$ supposes going on to a new solution Theses modifications are named movements. The binary variables have two possible values and the continuous variables are discretissed.

In every iteration, the best movement that is possible to realize from the current solution is chosen. In the Table I, the movement realized in each iteration is indicated with an arm in the corresponding variable. If the obtained solution is better than the previous ones it avoids. Unlike 
in the local search (that always searches the best of its environment and it finishes with a local optimum), the tabu search allows to move to worst solutions of the environment to avoid local minimum.

In the Table I also is observed that is prohibited to return to modify a parameter during a certain number of iterations (in this case during two iterations). The prohibited movements are stored in a named Tabu list.

In the Table I, the parameters that cannot be changed for belongs to the Taboo List are shaded. This mechanism has been observed usefully to escape of the local optimum and to continue strategically the search of even better solutions. The name of the heuristic method comes from this strategy.

The search continues until a stop criterion is fulfilled. Or, that the best reached solution does not improve in a certain number of iterations, or, the maximum number of iterations allowed is reached.

TABLE I. DEVELOPMENT OF TABU SEARCH.

\begin{tabular}{|c|c|c|c|c|c|c|c|c|c|c|}
\hline iter & $w_{1}$ & $w_{2}$ & $w_{3}$ & $w_{4}$ & $w_{5}$ & $\ldots$ & $w_{n-1}$ & $w_{n}$ & mov. & Tabu List \\
\hline 1 & & & & & & & & & $w_{2}$ & $\left\{w_{2}\right\}$ \\
\hline 2 & & & & & & & & & $w_{4}$ & $\left\{w_{4}, w_{2}\right\}$ \\
\hline 3 & & & & & 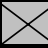 & & & & $w_{5}$ & $\left\{w_{5}, w_{4}, w_{2}\right\}$ \\
\hline 4 & & & & & & & & & $w_{2}$ & $\left\{w_{2}, w_{5}, w_{4}\right\}$ \\
\hline 5 & & & $x$ & & & & & & $w_{3}$ & $\left\{w_{3}, w_{2}, w_{5}\right\}$ \\
\hline
\end{tabular}

\section{Example case.}

\section{A. Description of the example.}

Figure 1 represents the studied case. It is necessary to highlight the following aspects: Node 0 is a bus balance and node 45 is a PV node. All nodes have connected an identical load. Dispersed generators are located in nodes 50 and 58. Lateral feeders from node 7 have inferior capacity. Feeders between the node 7 to node 39 represent rural netwok. Transformer 41-42 allows shiftphase regulation and transformer 16-17 have tap voltages. Capacitor bank is located in the node 33 .

There is wanted to construct a mini power central. The technology used to generate the above mentioned electricity can place with the same cost in the buses 9,12 , $14,16,17,20$ y 23.

\section{B. Reached solution and changes in the operation of the network.}

In the base case, the current in the feeders 7-8, 8-9, 9-10 and 10-11 exceeds a little the limit capacity. Before constructing the mini power central, the capacitor bank was supplied $16 \mathrm{kVAr}$ and the transformer 16-17 was in the maximum regulation 1,15 .

The algorithm connects and disconnects the generators, at the same time that it varies the rest of the control parameters. Trying to improve the current situation.

The evolution of the search is very rapid since it is possible to estimate in the Figure 2. The objective function reaches a value of 79,026 in 70 iterations.

The final result that one obtains is the placement of a new generator of 25 units of power in the bus 14 (see figure 1 ). There are not any limit capacity surpassed in the feeders, and the voltages in the nodes are inside of the limits.

The same optimization but without optimal operation allows to locate only a new generator of 16 units of power in the bus 14 . Besides the voltage in the bus 23 is lightly out of the allowed limits.

In the Figure 3, it is possible to observe the voltage profile of the netwok in all three cases: Case 0 (without the new generator and optimal operation), Case 1 (with the new generator and optimal operation), and Case 2 (with the new generator and without optimal operation).

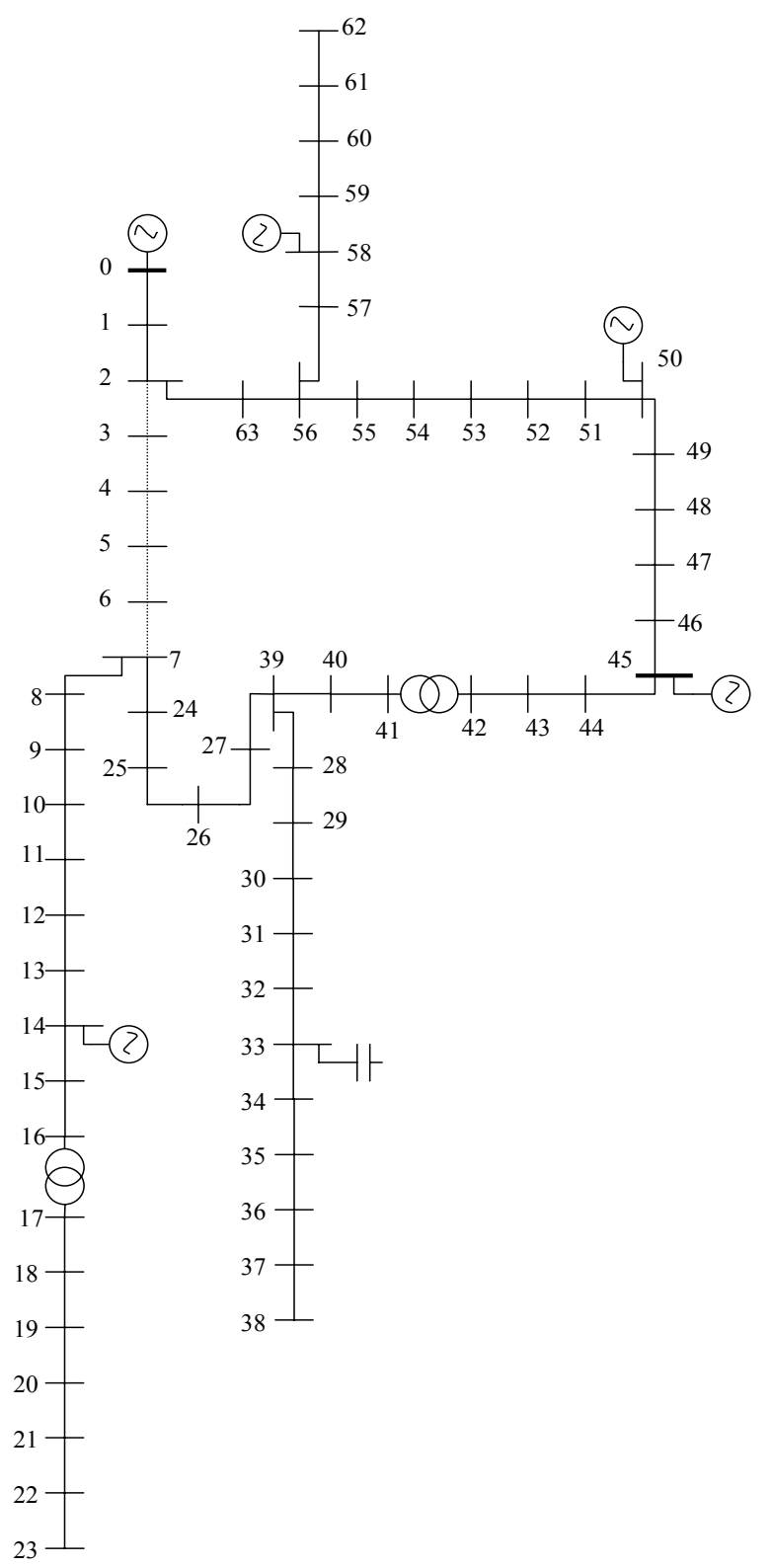

Figure 1. Example case.

\section{Conclusions and main contributions}

A search algorithm for the location of dispersed generator has been designed. The used algorithm is based on the heuristic method of tabu search. Improvements have been introduced to consider the modeling of dispersed 
generation (PV nodes), voltage regulators and regulated capacitor banks.

The object oriented programming is a potent tool for the modeling and simulation of electric systems, it allows the definition of general structures that are particularized to represent concrete devices.

The presented example demonstrates the validity and utility of the models and developed algorithms.

The obtained results clarify the importance of an optimal operation with distributed generation. On having considered the optimal operation in the location of new distributed generators has been observed that there can establish more generation (in the presented example the generated power has increased $56.25 \%$ ) and the voltage profile to improve considerably.

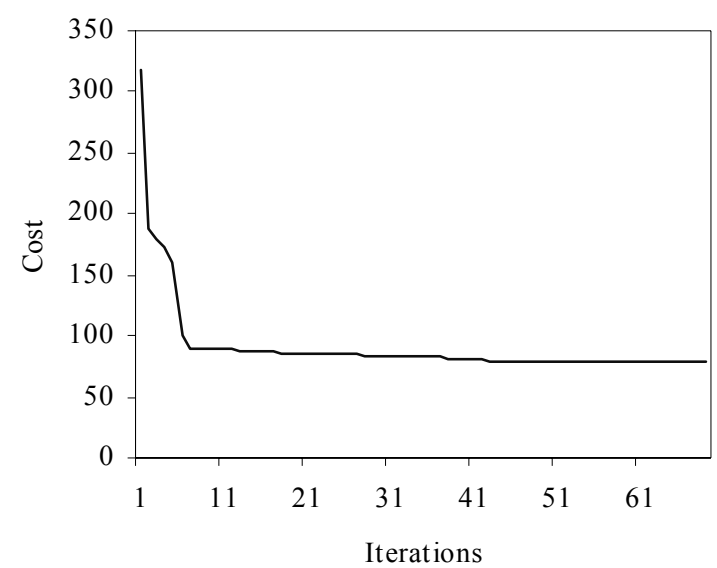

Figure 2. Evolution of the objective function.

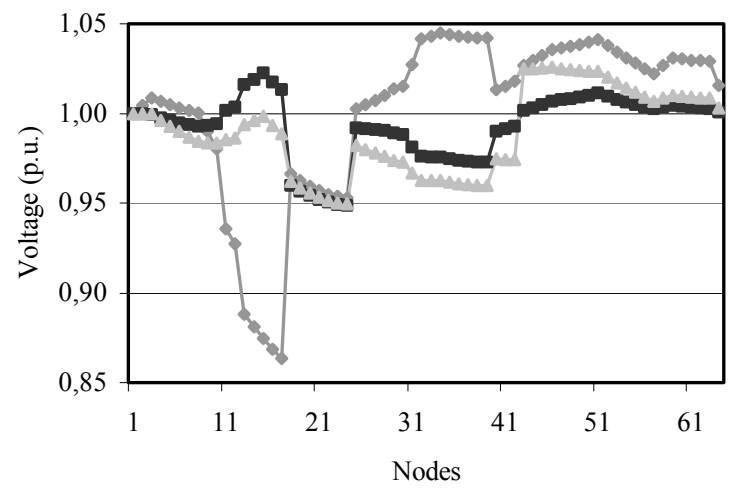

$\longrightarrow$ Case 0 (p.u.)) $\rightarrow-$ Case 1 (p.u.) $\longrightarrow$ Case 2 (p.u.)

Figure 3. Voltage in the nodes.

\section{References}

[1] W. El-Khattam, M.M.A. Salama, "Distributed generation technologies, definitions and benefits", Electric Power Systems Research, 71, 2004, 119-128.

[2] N.S. Rau, Y.H. Wan, "Optimum location of resources in distributed planning", IEEE Trans. Power Syst. 9 (4) (1994) 2014-2020.

[3] J.O. Kim, S.W. Nam, S.K. Park, C. Singh, "Dispersed generation planning using improved Hereford ranch algorithm", Electric Power Systems Research 47 (1998) 47-55.

[4] T. Ackermann, K. Garner, A. Gardiner, "Embedded wind generation in weak grids-economic optimisation and power quality simulation", Renewable Energy, 18, 1999, pp. 205-221.

[5] P. Dondi, D. Bayoumi, C. Haederli, D. Julian, M. Suter, "Network integration of distributed power generation", Journal of Power Sources 106 (2002) 1-9.

[6] A.S. Malik, C.U. Sumaoy, "Analytic hierarchy process approach in distribution utility local IRP", Electrical Power and Energy Systems, 25 (2003), 623-631.

[7] R. Raineri, S. Ríos, R. Vásquez, "Business opportunities and dynamic competition through distributed generation in primary electricity distribution networks", Energy Policy, article in press 2004.

[8] C. R. Reeves, "Heuristic Modern Techniques for Combinatorial Problems". New York: John Wiley \& Sons Inc., 1993. 